\title{
Distribution and evolution of high-z galaxies in the Subaru / XMM-Newton Deep Survey Field (SXDF)
}

\author{
K. Sekiguchi ${ }^{1}$ and M. Ouchi ${ }^{2}$ \\ ${ }^{1}$ Subaru Telescope, National Astronomical Observatory of Japan \\ ${ }^{2}$ Space Telescope Science Institute
}

\begin{abstract}
We present results from our on-going study of the distribution and evolution of highz galaxies. We exploited the large area coverage $\left(\sim 1.3 \mathrm{deg}^{2}\right)$ of the Subaru / XMM-Newton Deep Survey field (SXDF) to search for Lyman-break galaxies (LBGs) and Ly $\alpha$ Emitters (LAEs) at $3<z<7$. We have found filamentary large scale structures, which are made of LAEs, with $\sim 10$ to $40 \mathrm{Mpc}$ (in commoving units) scale voids as early as at $\mathrm{z}=5.7$. Galaxies at this redshift show clustering of $\sim 1 \mathrm{Mpc}$ in diameter (in physical unit). The inferred star formation rate density of these clustering is $\sim 130$ times the mean of the whole area, indicates burst of star formation activities. Combined with the UKIDSS/UDS photometry, the optical+near-IR SED fitting provides the estimate of masses for some of these high-z galaxies. Preliminary results indicate a number of galaxies at $\mathrm{z}>5$ have already grown up to $\sim 10^{11}$ solar mass.
\end{abstract}

Keywords. cosmology: large-scale structure, galaxies: distance and redshifts, evolution

\section{Discussion}

The Subaru / XMM-Newton Deep Survey field (Sekiguchi et al. 2004) has been a focus of our studies of the high redshift galaxies (Ouchi et al. 2005, Sekiguchi et al. 2006). The field has a deep multi-bands optical and near-infrared data set by Subaru Telescope and by the UKIRT WFCAM (Casali et al. 2006, in preparation) respectively, which can be exploited for selecting high-redshift galaxy candidates. The large area coverage of the SXDF resulted a discovery of structures by the LAEs and voids as early as at $\mathrm{z}=5.7$ (Ouchi et al. 2005). Also, with the early UKIDSS/UDS (Lawrence et al. 2006) data, McLure et al. (2006) found a few of the LBGs at $\mathrm{z}>5$ with the stellar mass up to $\sim 10^{11}$ solar mass.

\section{References}

Lawrence et al. 2006, MNRAS submitted, astro-ph/0604426

McLure, R. J. et al. 2006, MNRAS 372, 357

Ouchi, M. et al. 2005, ApJ (Letters) 620, L1

Sekiguchi, K. et al. 2004, Astrophisics and Space Science Library 301, 169

Sekiguchi, K. et al. 2006, in: P.A. Whitelock, M. Dennefeld \&/ B. Leibundgut (eds.), The Scientific Requirements for Extremery Large Telescopes, Proc. IAU Symp. 232, Cambridge Univ. Press, pp. 171-175 